\title{
Tratamiento quirúrgico del tórax inestable. đDónde nos encontramos? Experiencia de un hospital privado
}

\author{
Surgical treatment of the unstable thorax. Where do we stand? \\ A private hospital experience
}

Agustín Luna-Tovar,* María Rita Rodríguez Luna,** Rodrigo Morales Villalobos, ${ }^{*}$ Víctor Manuel Noriega Usi*

Palabras clave: Trauma de tórax, fracturas costales, tórax inestable, fijación quirúrgica, reconstrucción de la pared torácica.

Key words: Thoracic trauma, rib fractures, flail chest, surgical fixation, chest wall reconstruction.

* Cirujano General.

** Residente de Cirugía General.

Hospital Ángeles Mocel.

Recibido: $12 / 01 / 2016$

Aceptado: 30/11/2017

\section{RESUMEN}

Introducción: El trauma de tórax tiene implicaciones serias; representa el $20 \%$ de las muertes por trauma. El tórax inestable (TI), resultado de múltiples segmentos costales fracturados, agrava el problema y es responsable de una elevada morbilidad y mortalidad. Durante los últimos años, los pacientes se trataban con manejo del dolor y, en caso de requerirlo, estabilización neumática interna. En 1998, Tanaka y sus colaboradores compararon de forma prospectiva dicho manejo contra la estabilización quirúrgica; desde entonces, la evidencia científica ha mostrado resultados más favorables en pacientes que se someten a reducción abierta y fijación interna: se reduce la morbimortalidad, los días de estancia intrahospitalaria (EIH), los días de terapia intensiva con necesidad de ventilación mecánica y mejoran los resultados a largo plazo. Desde 2001, nuestro grupo inició la fijación de fracturas costales en pacientes con TI. Material y métodos: Este artículo muestra las distintas opciones que se han tenido para la estabilización quirúrgica a lo largo de los años; se presentan 11 casos de pacientes con diagnóstico de tórax inestable (TI), quienes fueron sometidos a manejo quirúrgico con fijación interna (RAFI) mediante sutura de alambre, sistemas de osteosíntesis maxilofacial y, en los últimos casos, dispositivos de titanio para fijación de fracturas como StraCos ${ }^{\circledR}$. Resultados: En 16 años, 10 pacientes presentaron trauma torácico cerrado y uno trauma torácico penetrante. Los pacientes tuvieron ISS promedio de 30.2 (DE =14.39). La media de EIH fue de 21.8 días $(\mathrm{DE}=11.14)$. La media de días de estancia en la unidad de cuidados intensivos (DEUCI) fue de 8.9 días $(\mathrm{DE}=4.89)$. El número de fracturas costales fijadas (NFCF) y los DEUCI mostraron una correlación negativa importante, $r(12)=-0.624, p=0.039$. A su vez, el NFC y los días en ventilación mecánica también mostraron una correlación negativa importante, $\mathrm{r}(12)=-0.698, \mathrm{p}=$ 0.015 , con el NFCF explicando el $48.7 \%$ de la variación encontrada en los días en ventilación mecánica (DVM). Se realizó una prueba t de muestra única analizando nuestros valores de DVM $(\mathrm{m}=6.45, \mathrm{DE}=4.61)$ contra los reportados en la literatura, sin encontrar diferencia significativa, $\mathrm{t}(10)=1.837, \mathrm{p}=0.096$. El número de fracturas costales

\section{ABSTRACT}

Introduction: Thoracic trauma has serious implications, accounting for $20 \%$ of all trauma deaths. Flail chest resulting from multiple rib fractures aggravates the problem and is responsible for significant morbidity and mortality. Initially, these patients were treated with pain management, and internal pneumatic stabilization when needed. In 1998, Tanaka and his group were the first to evaluate the differences between this management and surgical management with open reduction and internal fixation; since then, evidence has shown better outcomes with the surgical treatment, with a shorter hospital length of stay, less length of stay in the ICU with mechanical ventilation and better results. In 2001, our surgical team began the use of surgical rib fixation in patients with flail chest. Material and methods: This article evaluates the different surgical options for open reduction-internal fixation (ORIF) throughout time. We present 11 cases with a flail chest diagnosis that had surgical management. Initially, we used wire sutures for approximation and surgical fixation, osteosyntheses with maxillofacial devices and, lately, titanium devices such as traCos $^{\circledR}$. Results: During the past 16 years, ten patients presented to our hospital with closed thoracic trauma and one with penetrating trauma. The patients had a mean ISS of 30.2 $(S D=14.39)$. The mean hospital length of stay was 21.8 days $(S D=11.14)$. The mean of days in the intensive care unit $(D I C U)$ was 8.9 days $(S D=4.89)$. The number of rib fractures with internal fixation (NRFIF) and DICU showed an important negative correlation, $r(12)=-0.624, p=$ 0.039 . In turn, NRF and the days in mechanical ventilation (DMV) also showed an important negative correlation, $r$ (12) $=-0.698, p=0.015$, with the NRFIF explaining $48.7 \%$ of the variation found in the DMV. A single sample t test was performed, analyzing our DMV values ( $m=6.45, S D$ $=4.61$ ) against those reported in the literature, without finding significant difference, $t(10)=1.837, p=0.096$. The number of rib fractures treated with surgical fixation in patients was associated with a shorter stay in intensive care and less days of mechanical ventilation. This decrease was not found related to aggregated trauma and emergency 
tratadas con fijación quirúrgica en los pacientes se asoció con una menor estancia en terapia intensiva y menos días de ventilación mecánica. Esta disminución no se encontró relacionada con los traumatismos agregados y procedimientos quirúrgicos de urgencia a los que se sometieron dichos pacientes. Conclusiones: La fijación quirúrgica de fracturas costales mostró un beneficio significativo en la evolución clínica de los pacientes presentados. surgical procedures that these patients underwent. Conclusions: Surgical fixation of rib fractures showed a significant benefit in the clinical outcome of patients presented.

\section{INTRODUCCIÓN}

$\mathrm{E}^{\prime}$ trauma cerrado severo de tórax continúa siendo una de las principales causas de morbilidad y mortalidad en víctimas de trauma. Han surgido múltiples modalidades en los patrones de cuidado y tratamiento, muchas de ellas basadas tanto en la evidencia como en la observación clínica anecdótica. ${ }^{1}$

La fractura costal es la lesión esquelética más común en trauma cerrado de tórax y ocurre aproximadamente en el 50\% de los pacientes. Las fracturas de la primera a la tercera costilla son un marcador para trauma de alta velocidad por estar protegidas por la clavícula y la musculatura de la pared torácica superior. Las fracturas de la octava a la décimo primera costilla deberían ser evaluadas rápida y cuidadosamente debido a su asociación con lesiones de órganos intraabdominales superiores. Los pacientes que tienen fracturas costales derechas a este nivel tienen de 19 a 56\% de probabilidad de lesión hepática, mientras que aquéllos con lesión del lado izquierdo tienen de 22 a 28\% de probabilidad de lesión esplénica. ${ }^{2}$

El tórax inestable (TI) se produce cuando hay al menos dos sitios de fractura en tres o más costillas consecutivas, lo que resulta en un segmento libre flotante de costillas y provoca inestabilidad en la coordinación de la pared torácica en su mecánica respiratoria. ${ }^{3-5}$

El movimiento paradójico del segmento fracturado altera la dinámica pulmonar normal y produce atelectasias, estasis de las secreciones y neumonía. ${ }^{4}$ Esta condición se observa en 5 a $13 \%$ de los pacientes con traumatismo de tórax. ${ }^{3}$

EI TI se produce en 1\% de los traumas torácicos cerrados; tiene una mortalidad reportada del 16\%; 6 la morbilidad está directamente relacionada con el número de fracturas y la edad del paciente, y puede ser tan alta como
33 a 35\%. 3,7 La mayoría de los TI presentan dolor crónico incluso hasta 30 días posteriores a la lesión inicial, y requieren altas dosis de analgésicos. ${ }^{8} \mathrm{El}$ riesgo de muerte disminuye un $40 \%$ con un adecuado control analgésico. ${ }^{7}$

Por estas razones, los cirujanos hemos investigado la posibilidad de mejorar los resultados en estos pacientes mediante la fijación interna quirúrgica de las costillas y corrección de la deformidad condicionada por un tórax inestable. Existen prótesis diseñadas específicamente para este propósito. Las placas metálicas, alambres de Kirschner, clavos y alambres envolventes han sido ensayados todos, pero sin éxito suficiente como para llevar a la amplia adopción de esas técnicas, que actualmente se encuentran en desuso. $^{9}$

Una piedra angular que permitió la evolución del manejo en la actualidad de pacientes con $\mathrm{TI}$ fue el estudio realizado por Tanaka y sus colaboradores en 1998 y publicado en The Journal of Trauma, un estudio prospectivo que comparaba la eficacia clínica de la estabilización quirúrgica de las fracturas costales en pacientes con tórax inestable severo con requerimiento de ventilación mecánica prolongada contra la clásica estabilización neumática interna popularizada desde 1960; sus resultados afirmaron que los pacientes sometidos a manejo quirúrgico presentan disminución en la morbilidad y en costos médicos, menor incidencia de neumonía y un tiempo de intubación más breve. ${ }^{10}$

Con el advenimiento de múltiples estudios de alta calidad científica, publicados en los últimos 16 años, la fijación quirúrgica ha ganado popularidad progresiva. Esto demuestra que la estabilización quirúrgica debe ser indicada en el tratamiento del tórax inestable debido a sus ventajas, tales como la disminución en la estancia hospitalaria $(\mathrm{EIH})$ y complicaciones 
pulmonares; adicionalmente, la estabilización quirúrgica de las fracturas costales puede reducir el dolor, disminuir los días en ventilación mecánica (DMV) y, por tanto, los días de estancia en terapia intensiva (DEUCI). ${ }^{1,11,12}$

A pesar de los beneficios encontrados, a la fecha no existen indicaciones específicas de fijación quirúrgica para pacientes con fracturas costales, y se ha observado que a pesar de que las ventajas son ampliamente reconocidas por los cirujanos, sólo el $26 \%$ de los cirujanos de trauma realizan la reducción quirúrgica. ${ }^{7}$

Existen dos estudios nivel uno en los cuales se compara el tratamiento quirúrgico contra el conservador; ambos demostraron menos DEUCl y EIH. ${ }^{12}$

Las placas costales no son de aparición reciente, pero la utilización de titanio para su construcción sí lo es; el titanio tiene biocompatibilidad con los tejidos, lo que lo hace ideal para prótesis a largo plazo, pues permanece inerte y libre de corrosión con el paso de los años. El titanio, a diferencia del acero, es más flexible, se adapta con exactitud al arco costal y no presenta tensión sobre el sitio de fractura, lo que lo convierte en un material ideal. En la actualidad, es ampliamente utilizado en los dispositivos de fijación costal. ${ }^{8}$

\section{MATERIAL Y MÉTODOS}

Presentamos 11 pacientes que fueron operados en el Hospital Ángeles Mocel de la Ciudad de México, de 2001 a 2010. Los criterios de selección para la estabilización quirúrgica de fracturas costales fueron pacientes con trauma cerrado y/o penetrante de tórax, con tórax inestable y:

- Dolor significativo asociado al movimiento de las fracturas costales, con difícil control a pesar del manejo analgésico combinado (antiinflamatorio no esteroideo y opioide).

- Defectos y/o deformidades en la pared torácica que comprometieran la mecánica ventilatoria.

- Tórax inestable con o sin ventilación mecánica invasiva.

El manejo quirúrgico ofrecido fue reducción abierta y fijación interna mediante cerclaje con alambre quirúrgico (Figura 1), miniplacas de titanio de cirugía maxilofacial y barras de titanio StraCos ${ }^{\circledR}$ (Figuras 2 y 3) (Strasbourg Costal Osteosyntheses System, MedXpert, Heitersheim, Germany) en los últimos años (Figuras 4 y 5). Todos los pacientes fueron manejados inicialmente de acuerdo con los criterios del curso ATLS (Advanced Trauma Life Support) del Colegio Americano de Cirujanos.

La tomografía axial computada multicorte (TAC) fue usada para descartar la presencia de hemotórax, neumotórax y contusión pulmonar, y evaluar los trazos de fractura; se hicieron reconstrucciones en 3D para la planeación quirúrgica (Figuras 6 y 7 ).

Se les realizó reducción abierta y fijación interna de las fracturas costales utilizando diferentes métodos. Técnica quirúrgica bajo intubación orotraqueal. Dependiendo de la localización de las fracturas, se seleccionó la posición del paciente, así como el tipo de incisión; en al menos un caso se utilizó la posición de decúbito dorsal. Se expusieron las fracturas, desperiostizando el sitio fracturado y evitando lo más posible la disección completa del músculo intercostal; siempre se trató de hacer un abordaje extrapleural; en algunos casos hubo necesidad de entrar a cavidad pleural para drenaje del hemotórax y/o reparar lesiones intratorácicas asociadas.

Tres pacientes fueron sometidos a osteosíntesis con miniplacas de titanio de cirugía maxilofacial, a tres se les fijaron las fracturas costales con cerclaje de alambre quirúrgico,

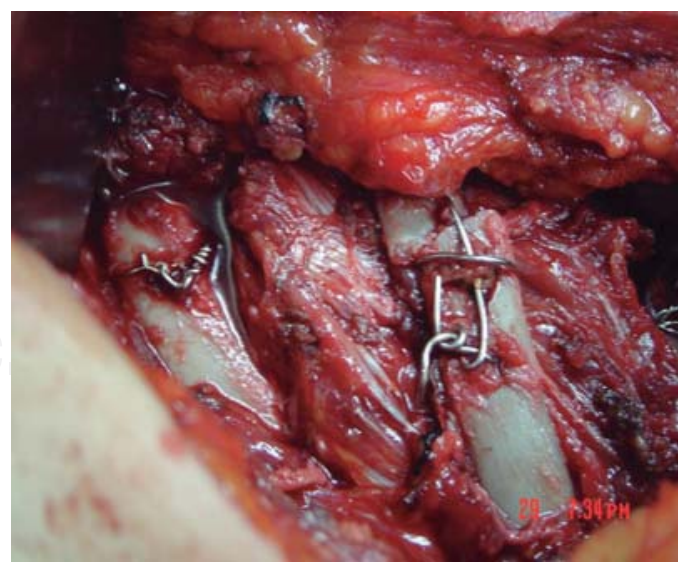

Figura 1. Cerclaje con sutura de acero. Se observa desperiostizada sólo la zona afectada. 
en uno se usó sutura de alambre quirúrgico y en cinco se utilizaron grapas costales de titanio StraCos ${ }^{\circledR}$ para la fijación.

Después de la fijación de las fracturas costales, se cerró la pared por planos, dejando un drenaje cerrado subcutáneo, así como una sonda endopleural. Posteriormente, pasaron a la unidad de terapia intensiva.

\section{RESULTADOS}

A lo largo del estudio, 10 pacientes presentaron trauma torácico cerrado y uno trauma torácico penetrante. Los pacientes tuvieron ISS promedio de 30.2 (DE =14.39). La ElH media fue de 21.8 días $(\mathrm{DE}=11.14)$. La media de DEUCl fue de 8.9 días ( $D E=4.89$ ); ningún paciente requirió de traqueostomía por intubación orotraqueal prolongada. Las características de los pacientes se enumeran en el cuadro $I$.

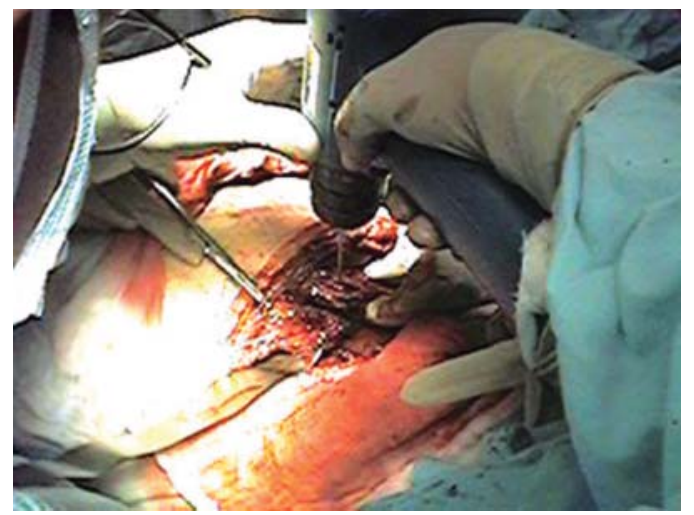

Figura 2. Fijación del material de osteosintesis.

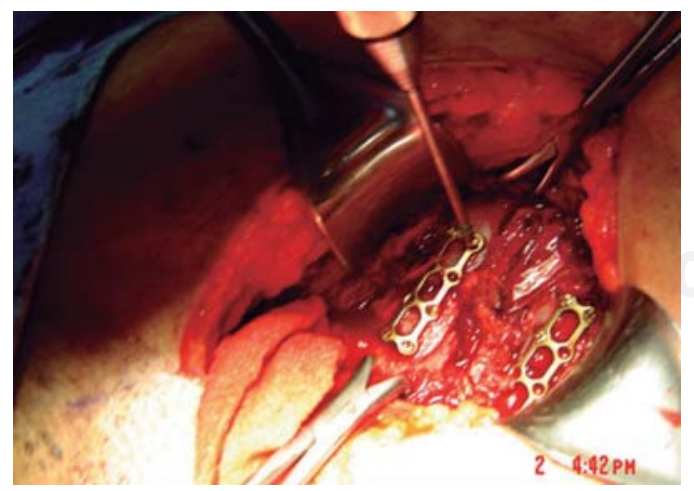

Figura 3. Fijación del material de osteosintesis, placas de cirugía maxilofacial.
Un paciente presentó empiema (8\%), por lo que se tuvo que someter a toracotomía para lavado y decorticación. Cabe notar que este paciente presentaba un alto riesgo de complicación por presencia de hemotórax retenido de 20 días de evolución, siendo éste el paciente con la EIH más alta. Un paciente presentó neumonía, la cual se manejó con carbapenémico y aminoglucósido. Se requirieron procedimientos quirúrgicos adicionales en nueve (75\%) casos. Un paciente requirió hepatorrafia por trauma hepático grado III y reparo de desgarros en la raíz del mesenterio; uno precisó ventana pericárdica por presencia de tamponamiento cardiaco con repercusión hemodinámica y colapso de las cavidades derechas por ecocardiograma transtorácico (ECOTT); uno más requirió de esplenectomía por lesión esplénica grado III y otro necesitó segmentectomía pulmonar por lesión penetrante con avulsión de los segmentos basal medial y basal lateral del lóbulo inferior del pulmón izquierdo. Los diferentes traumatismos agregados a las fracturas costales, cirugías de urgencia y complicaciones se enumeran en el cuadro II.

La estadística inferencial fue analizada en el programa SPSS 23 (IBM) por uno de los autores (RLMR).

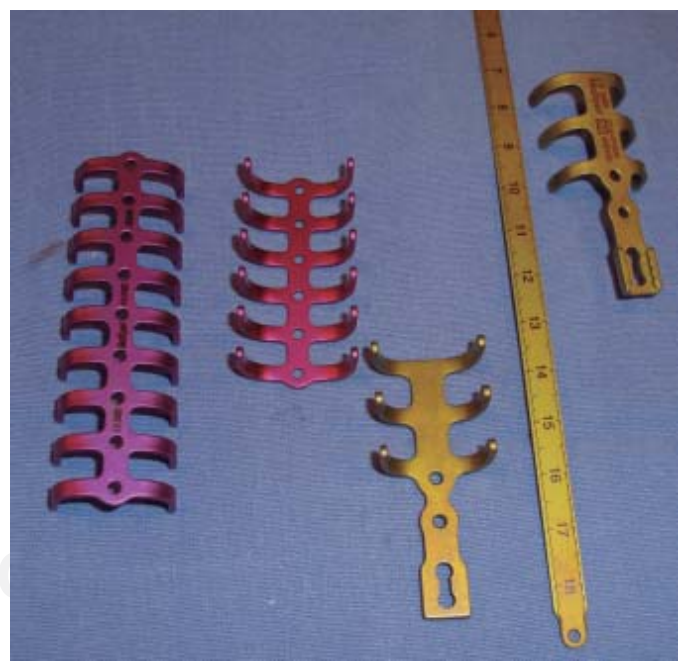

Figura 4. Material de fijación costal StraCos ${ }^{\circledR}$ (Strasbourg Costal Osteosyntheses System, MedXpert, Heitersheim, Germany). De izquierda a derecha, clip costal de nueve segmentos, clip costal de seis segmentos, clip de tres segmentos y barra conectora de $190 \mathrm{~mm}$. 

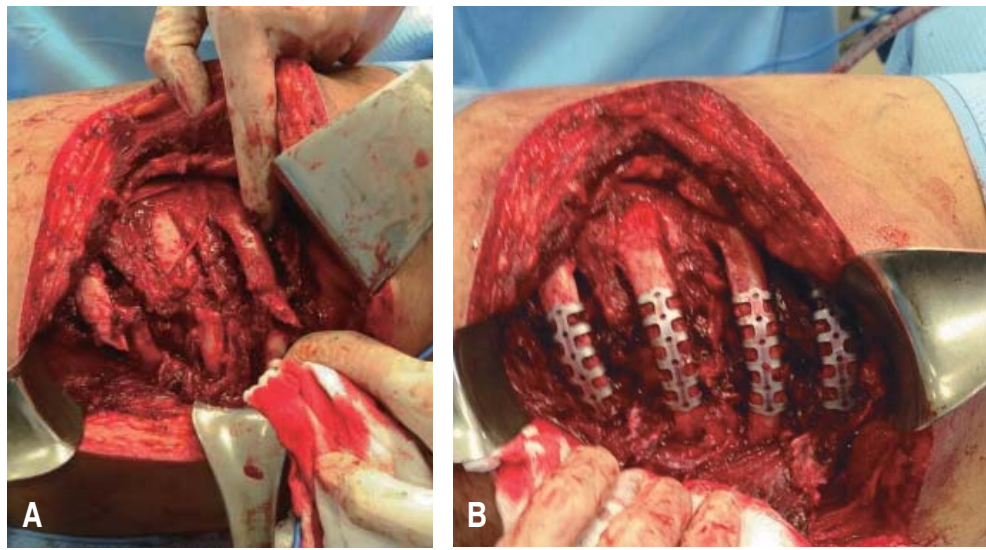

Figura 5. Visión intraoperatoria. A) Reducción abierta. B) Fijación interna con clips de titanio StraCos ${ }^{\circledR}$.

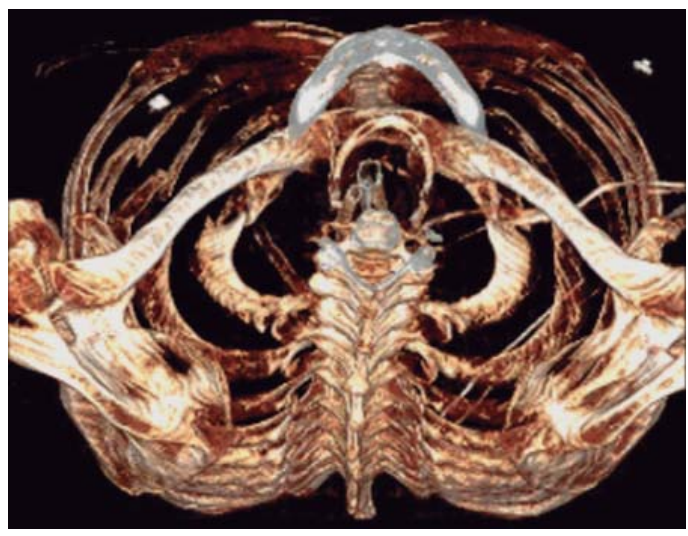

Figura 6.

Reconstrucción tomográfica en $3 D$. Corte axial a nivel del opérculo torácico, presencia de rotoescoliosis y fracturas de arcos anteriores izquierdos.
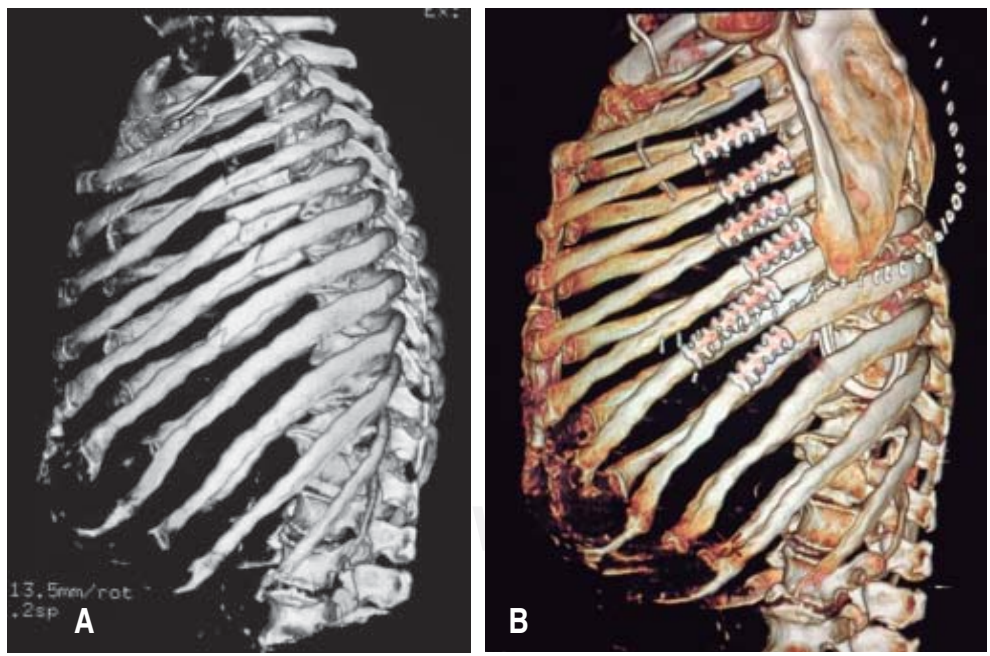

Figura 7. TAC de tórax, reconstrucción 3D. A) Fracturas costales laterales del segundo al séptimo arco costal izquierdo desplazadas; utilizada para la planeación quirúrgica. B) Posteriormente a RAFI con clips de titanio, permite evaluar la adecuada fijación del material de osteosíntesis.
Una correlación producto-momento de Pearson se llevó a cabo para evaluar la relación entre el número de fracturas costales estabilizadas quirúrgicamente (NFC) y la estancia en terapia intensiva e intrahospitalaria. Los análisis preliminares mostraron una relación lineal con las variables distribuidas normalmente, evaluadas mediante la prueba de Shapiro-Wilk ( $p>0.05)$, sin evidencia de valores atípicos. El NFC y los DEUCI mostraron una correlación negativa importante, $r(12)=-0.624$, $p=0.039$, con el NFC explicando el $38.9 \%$ de la variación encontrada en la estancia en terapia intensiva. A su vez, el NFC y los días en ventilación mecánica también mostraron una correlación negativa importante, $r(12)=$ -0.698, $p=0.015$, con el NFC explicando el $48.7 \%$ de la variación encontrada en los días en ventilación mecánica. Se muestra el resultado obtenido con las variables del estudio en el cuadro III.

Hubo siete (58\%) pacientes que necesitaron cirugía urgente por lesiones torácicas y abdominales agregadas al trauma costal. Se llevó a cabo una prueba para muestras independientes t-test para determinar si había diferencias en los ElH, DEUCl y DVM entre ambos grupos. No hubo valores atípicos en los datos, tal como se evaluó mediante la inspección de un diagrama de cajas y bigotes. La distribución para los dos grupos fue normal, tal como se evaluó mediante la prueba de Shapiro-Wilk ( $p>0.05$ ), y hubo una homogeneidad de las varianzas distintas en las variables, tal como se valoró por el test de Levene, que se muestra en el cuadro II. No hubo diferencias significativas en la prueba de muestras independientes, como se aprecia en el cuadro IV. No hubo mortalidad en nuestra serie $(0 \%)$.

Se realizó una prueba t de muestra única analizando nuestros valores de DVM ( $\mathrm{m}=$ $6.45, \mathrm{DE}=4.61$ ) contra los reportados en la literatura, ${ }^{13}$ sin encontrar diferencia significativa, $\mathrm{t}(10)=1.837, \mathrm{p}=0.096$.

\section{DISCUSIÓN}

En esta revisión se trataron 11 pacientes con fijación quirúrgica de las fracturas de arcos costales utilizando diferentes métodos, los cuales facilitaron el destete del apoyo ventila- 
Cuadro I. Pacientes contenidos en el presente estudio, junto con las diferentes variables estudiadas.

\begin{tabular}{clllllllll} 
Paciente & Edad & NFC & ISS & RTS & TRISS & DEIH & DEUCI & DVM & CTS \\
\hline P1 & 46 & 3 & 50 & 6.9 & 72.2 & 16 & 10 & 9 & 7 \\
P2 & 65 & 4 & 50 & 7.84 & 49.3 & 28 & 14 & 12 & 9 \\
P3 & 60 & 4 & 27 & 6.37 & 67 & 22 & 17 & 12 & 10 \\
P4 & 77 & 3 & 25 & 7.84 & 88.7 & 11 & 9 & 7 & 9 \\
P5 & 49 & 4 & 16 & 7.84 & 99 & 17 & 7 & 5 & 9 \\
P6 & 25 & 5 & 44 & 7.84 & 90.2 & 25 & 10 & 8 & 8 \\
P7 & 46 & 5 & 24 & 7.1 & 96.4 & 29 & 12 & 10 & 9 \\
P8 & 63 & 7 & 18 & 7.84 & 91.7 & 49 & 4 & 0 & 7 \\
P9 & 71 & 7 & 6 & 7.84 & 97.5 & 9 & 0 & 0 & 6 \\
P10 & 50 & 6 & 38 & 5.97 & 71.59 & 20 & 11 & 8 & 7 \\
P11 & 67 & 6 & 35 & 7.84 & 77.3 & 14 & 4 & 0 & 9 \\
Media & 56.27 & 4.91 & 30.27 & 7.38 & 81.9 & 21.82 & 8.91 & 6.45 & 8.18 \\
DE & 14.75 & 1.44 & 14.39 & 0.69 & 15.7 & 11.14 & 4.89 & 4.61 & 1.25 \\
\hline
\end{tabular}

$\mathrm{NFC}$ = número de fracturas costales fijadas; ISS = Injury Severity Score; RTS = Revised Trauma Score; DEIH = días de estancia intrahospitalaria; DEUCI = días de estancia en la unidad de cuidados intensivos; DVM = días de ventilación mecánica; $\mathrm{CTS}=$ Chest Trauma Score; Media = media; $\mathrm{DE}=$ desviación estándar.

\section{Cuadro II. Traumatismos agregados, procedimientos adicionales y complicaciones.}

Paciente Complicaciones pulmonares Cirugía adicional

\begin{tabular}{ccc}
\hline P1 & No & \multicolumn{2}{c}{ Hepatorrafia } \\
P2 & No & $\begin{array}{c}\text { Empaquetamiento } \\
\text { Osteosíntesis, fracturas } \\
\text { hepático }\end{array}$ \\
miembros pélvicos
\end{tabular}

torio; todos fueron dados de alta del hospital sin tener mortalidad. Los resultados fueron independientes de los traumatismos asociados y los procedimientos quirúrgicos de urgencia agregados a los que fueron sometidos.

La Eastern Association for the Surgery of Trauma, en las guías de práctica para el manejo de tórax inestable, apoya el uso del tratamiento quirúrgico con fijación de fracturas de costales en aquellos pacientes con imposibilidad para progresar la ventilación mecánica y aquéllos con difícil control analgésico, considerando la anestesia regional. ${ }^{7}$ Otras indicaciones son tórax inestable, deformidad de la caja 


\begin{tabular}{|c|c|c|c|c|c|c|c|c|}
\hline & & $\mathrm{NCF}$ & DEUCI & ISS & RTS & TRISS & DEIH & DVM \\
\hline \multirow[t]{2}{*}{ NCF } & Correlación de Pearson & 1 & $-.624 *$ & -0.441 & 0.102 & 0.318 & 0.315 & $-.698^{*}$ \\
\hline & Sig. (bilateral) & & 0.04 & 0.175 & 0.765 & 0.341 & 0.345 & 0.017 \\
\hline \multirow[t]{2}{*}{ DEUCI } & Correlación de Pearson & $-.624^{*}$ & 1 & 0.551 & -0.565 & -0.601 & 0.139 & $.956 * *$ \\
\hline & Sig. (bilateral) & 0.04 & & 0.079 & 0.07 & 0.05 & 0.683 & 0 \\
\hline \multirow[t]{2}{*}{ ISS } & Correlación de Pearson & -0.441 & 0.551 & 1 & -0.236 & $-.728^{*}$ & 0.036 & 0.566 \\
\hline & Sig. (bilateral) & 0.175 & 0.079 & & 0.484 & 0.011 & 0.917 & 0.069 \\
\hline \multirow[t]{2}{*}{ RTS } & Correlación de Pearson & 0.102 & -0.565 & -0.236 & 1 & 0.364 & 0.043 & -0.503 \\
\hline & Sig. (bilateral) & 0.765 & 0.07 & 0.484 & & 0.271 & 0.901 & 0.115 \\
\hline \multirow[t]{2}{*}{ TRISS } & Correlación de Pearson & 0.318 & -0.601 & $-.728^{*}$ & 0.364 & 1 & -0.03 & -0.546 \\
\hline & Sig. (bilateral) & 0.341 & 0.05 & 0.011 & 0.271 & & 0.931 & 0.082 \\
\hline \multirow[t]{2}{*}{ DEIH } & Correlación de Pearson & 0.315 & 0.139 & 0.036 & 0.043 & -0.30 & 1 & 0.013 \\
\hline & Sig. (bilateral) & 0.345 & 0.683 & 0.917 & 0.901 & 0.931 & & 0.969 \\
\hline \multirow[t]{2}{*}{ DVM } & Correlación de Pearson & $-.698^{*}$ & $.956^{* *}$ & 0.566 & -0.503 & -0.546 & 0.013 & 1 \\
\hline & Sig. (bilateral) & 0.017 & 0 & 0.069 & 0.115 & 0.082 & 0.969 & \\
\hline
\end{tabular}

* La correlación es significativa en el nivel 0.05 (bilateral). ** La correlación es significativa en el nivel 0.01 (bilateral).

$\mathrm{NCF}$ = número de costillas fijadas; ISS = Injury Severity Score; RTS = Revised Trauma Score; DEIH = días de estancia intrahospitalaria; DEUCI

= días de estancia en la unidad de cuidados intensivos; $\mathrm{DVM}=$ días con ventilación mecánica; $\mathrm{CP}=$ complicaciones pulmonares; $C T S=$ Chest

Trauma Score; $\mathrm{CA}=$ cirugía adicional.

\begin{tabular}{|c|c|c|c|c|c|c|c|}
\hline \multicolumn{8}{|c|}{$\begin{array}{l}\text { Cuadro IV. Prueba de muestras independientes para } \\
\text { presencia } 0 \text { ausencia de lesiones traumáticas agregadas. }\end{array}$} \\
\hline & & \multicolumn{2}{|c|}{$\begin{array}{c}\text { Prueba de Levene } \\
\text { de igualdad de } \\
\text { varianzas }\end{array}$} & \multicolumn{3}{|c|}{$\begin{array}{l}\text { Prueba t para la igualdad } \\
\text { de medias }\end{array}$} & \\
\hline & & \multirow[t]{2}{*}{$\mathrm{F}$} & \multirow[t]{2}{*}{ Sig. } & \multirow[t]{2}{*}{$\mathrm{t}$} & \multirow[t]{2}{*}{$\begin{array}{l}\text { Sig. } \\
\text { (bilateral) }\end{array}$} & \multicolumn{2}{|c|}{$\begin{array}{c}95 \% \text { de intervalo } \\
\text { de confianza de la } \\
\text { diferencia }\end{array}$} \\
\hline & & & & & & Inferior & Superior \\
\hline \multirow[t]{2}{*}{ DEIH } & Se asumen varianzas iguales & 2.299 & 0.164 & 0.697 & 0.503 & -11.222 & 21.222 \\
\hline & No se asumen varianzas iguales & & & 0.56 & 0.608 & -20.798 & 30.798 \\
\hline \multirow[t]{2}{*}{ DEIUCI } & Se asumen varianzas iguales & 5.988 & 0.037 & -0.447 & 0.665 & -8.654 & 5.797 \\
\hline & No se asumen varianzas iguales & & & -0.361 & 0.738 & -12.844 & 9.987 \\
\hline \multirow[t]{2}{*}{ DVM } & Se asumen varianzas iguales & 2.488 & 0.149 & -0.775 & 0.458 & -8.96 & 4.389 \\
\hline & No se asumen varianzas iguales & & & -0.684 & 0.528 & -11.178 & 6.607 \\
\hline
\end{tabular}

$\mathrm{DEIH}$ = días de estancia intrahospitalaria; DEIUCI = días estancia intrahospitalaria en unidad de cuidados intensivos; DVM = días de ventilación mecánica. 
torácica y fracturas desplazadas que lesionan el parénquima pulmonar, así como fracturas expuestas. ${ }^{3,4,14}$

En la primera mitad de los años 90, el abordaje de las lesiones torácicas severas incluía manejo no quirúrgico como posicionamientos y tracción externa de los segmentos fracturados, lo que permitía una limitación del movimiento paradójico; sin embargo, condicionaba al paciente a una posición supina prolongada. Los reportes de fijación interna aparecieron en 1950; dentro de las técnicas empleadas incluían la sutura con alambre. La estabilización interna con presión positiva se volvió popular; sin embargo, las complicaciones secundarias e infecciones debido a la ventilación mecánica prolongada condicionaban una mortalidad del 10 al 36\%, lo que condujo a buscar nuevamente una ventaja con una fijación interna temprana. ${ }^{15}$ Tanaka y sus colaboradores, en 1998, compararon de forma prospectiva en 37 pacientes las ventajas de una estabilización quirúrgica; encontraron disminución en la morbilidad y en costos médicos, menor incidencia de neumonía y menor tiempo de intubación.

La estabilización neumática con un flujo de aire a presión en la vía aérea la mantiene abierta y expedita. La presión continua positiva de la vía aérea (CPAP) ha sido el manejo para pacientes con movimiento paradójico marcado de la pared torácica que provoca compromiso respiratorio; se les ha llevado a periodos de ventilación prolongada con resultados pobres a largo plazo, como síndrome de dolor crónico, incapacidad para regresar al trabajo, restricción del movimiento de la pared torácica y deformidad torácica, con la consiguiente disminución de las capacidades pulmonares. ${ }^{16}$

La reparación de fracturas costales se ha realizado en centros seleccionados de todo el mundo desde hace más de 50 años; sin embargo, las indicaciones quirúrgicas no han sido establecidas y son objeto de polémica. El resultado de un enfoque estrictamente conservador puede no ser óptimo. Las indicaciones potenciales para la reparación de fracturas costales incluyen tórax inestable, dolor, fracturas costales móviles refractarias al tratamiento convencional del dolor, deformidad o defecto de la pared torácica, fracturas costales separadas y durante una toracotomía por otra indicación traumática. La reparación de fracturas costales es técnicamente difícil debido a que las costillas humanas tienen una corteza relativamente delgada y tendencia a la fracturas oblicuas. Sin embargo, se han desarrollado varios sistemas eficaces de reparación. Orientaciones futuras para el progreso en este importante problema quirúrgico incluyen el desarrollo de técnicas mínimamente invasivas para el tratamiento y la realización de estudios multicéntricos, aleatorizados. ${ }^{17}$

En un trabajo de Velmahos y su grupo, el tórax inestable se relaciona con mayor morbilidad y requiere de intubación temprana. Se conformaron dos grupos de estudio, el primero de 22 pacientes, todos ellos con tórax inestable, y el segundo formado por 68 pacientes con fracturas costales, pero sin tórax inestable. En este estudio, predicen mayor frecuencia de complicaciones pulmonares en forma independiente el tórax inestable y las lesiones extratorácicas. Así, $86 \%$ de los pacientes con tórax inestable requirieron intubación, contra $46 \%$ del grupo control. Desarrollaron neumonía y síndrome de insuficiencia respiratoria del adulto (SIRA) $64 \%$ de los pacientes con tórax inestable contra $42 \%$ del grupo control. Los pacientes con tórax inestable requirieron ventilación mecánica por periodos mayores a una semana. Velmahos y sus colegas concluyeron que la inestabilidad de la caja torácica parece ser la causa de la dificultad respiratoria, por lo que estos pacientes se deben intubar en forma temprana y liberal, sobre todo cuando tienen lesiones extratorácicas asociadas, dado que, en su experiencia, los retrasos en la intubación de estos pacientes se correlacionan con mayor índice de atelectasias y mayor frecuencia de infección intratorácica y SIRA. ${ }^{18}$

En nuestra serie se ha intentado dar manejo con ventilación mecánica no invasiva a nueve de 11 (81.81\%) pacientes; todos ellos han sido manejados en las áreas de cuidados intermedios y terapia intensiva, en donde se han monitorizado en forma continua, $y$ ante el menor dato de deterioro, fueron sometidos a intubación orotraqueal y ventilación mecánica invasiva. Uno de ellos no fue sometido a intubación orotraqueal sino hasta el momento de la cirugía torácica; uno más fue intubado debido al deterioro neurológico con monoparesia, así, fue llevado a TAC de cráneo con asistencia de ventilación mecánica y en compañía de un 
anestesiólogo del hospital; los restantes tres pacientes fueron intubados debido a hipoxemia $\left(\mathrm{PaO}_{2}<60\right)$ detectada por gasometría arterial. Consideramos que el alto índice de intubación de nuestra serie se ha debido a contusión pulmonar y a la presencia de lesiones extratorácicas y/o patologías asociadas.

Ahmed y Mohyuddin documentan en su trabajo que pacientes con fijación quirúrgica permanecen en promedio 3.9 días en ventilador, contra 15 días para pacientes sin cirugía; ${ }^{13}$ estos valores no difieren significativamente de nuestra serie.

Datos similares son reportados por Voggenreiter, quien registró menor tiempo de ventilación mecánica en pacientes con fijación interna (quirúrgica) comparados con aquéllos con estabilización neumática (6.5 días para el primer grupo contra 26.7 días para el último grupo), siempre y cuando los pacientes no tuvieran, además contusión pulmonar. Pacientes con TI y dificultad respiratoria sin contusión pulmonar se benefician de la fijación de los arcos costales. Pacientes con TI y contusión pulmonar no se benefician de la fijación de los arcos costales y deberá reservarse sólo para aquellos casos en que se detecte movimiento paradójico al intentar retirarlos del ventilador. ${ }^{19}$

El tiempo ideal para realizar el procedimiento quirúrgico no está establecido; sin embargo, se ha observado que un manejo quirúrgico temprano reduce la estancia en la unidad de cuidados intensivos, así como la estancia intrahospitalaria. Fowler y sus colaboradores recomiendan una fijación dentro de los primeros tres a cinco días de la lesión inicial. ${ }^{3}$

Tanaka introduce al manejo presión soporte en ventilación espontánea, CPAP (presión positiva continua en la vía aérea), con mascarilla y terapia respiratoria por terapeutas físicos. ${ }^{20}$ Todos nuestros pacientes recibieron en forma temprana apoyo por medicina física y por el servicio de apoyo respiratorio; con frecuencia fueron atendidos por ambos terapeutas al mismo tiempo para reeducación funcional, terapia oscilatoria y terapia con CPAP. Consideramos que ello contribuyó a disminuir las complicaciones y aumentar la supervivencia de nuestros pacientes.

Hatem Alkadhi y su grupo en Suiza efectuaron un estudio en el que incluyó un total de 30 pacientes, de un grupo de 50 en quienes se encontró un total de 178 fracturas costales; en su trabajo evaluaron la precisión diagnóstica para las imágenes de TAC convencionales (TAC con imágenes transversas) contra las imágenes de tipo 3D, sensibilidad, especificidad y precisión diagnóstica: TC transversa multidetector: 96, 100 y 99\%, imágenes 3D: 98, 100 y 100\%. Los resultados hacen comparables a ambos métodos; sin embargo, las imágenes tipo 3D parecen leerse más rápidamente y tienen la ventaja de mostrar al mismo tiempo varios arcos costales, lo mismo que el esternón; además, se pueden girar en diferentes sentidos y ángulos, permitiendo con ello crear una idea clara del total de fracturas y el sitio donde se ubican, lo que nos ayuda a planear el mejor abordaje. ${ }^{21}$ La tomografía computada con reconstrucción en tercera dimensión (3D) fue utilizada en cada uno de los pacientes como evaluación preoperatoria y planeación quirúrgica.

\section{CONCLUSIONES}

El TI es una entidad grave, con morbilidad y mortalidad elevadas. La insuficiencia respiratoria está dada, al menos en parte, por la alteración de la dinámica de la pared torácica y el dolor secundario. El TI continúa siendo asociado con una morbimortalidad importante. El tratamiento quirúrgico puede mejorar la dinámica torácica y disminuir el dolor.

La fijación de los arcos costales disminuye el tiempo de ventilación mecánica, la estancia en la UTA y el riesgo de neumonía. Parece ser que el mejor momento para efectuar la cirugía es entre el segundo y el cuarto día de estancia intrahospitalaria (antes de que se desarrollen complicaciones como atelectasias y neumonía), lo que condiciona que un tratamiento quirúrgico temprano aumente las posibilidades de sobrevivir.

Las imágenes de tomografías de tórax con reconstrucciones en 3D nos permiten ventajas en la planeación quirúrgica para evaluar los arcos costales a tratar y el tipo de incisión que permitirá la adecuada exposición. El tratamiento quirúrgico puede y debe individualizarse y adaptarse a las necesidades de cada paciente.

La fijación quirúrgica de las fracturas costales se indica no sólo en el trauma torácico cerrado, sino también en el trauma penetrante con deformidad y pérdida de los componentes 
de la pared torácica. Ayuda a disminuir la insuficiencia respiratoria, los días de ventilación mecánica, la mortalidad y complicaciones asociadas con la intubación prolongada. Dos metaanálisis recientes identifican resultados clínicos superiores cuando se utiliza la reducción abierta y fijación interna de fracturas de costillas, y respaldan su utilización. ${ }^{11,22}$

Nuestro hospital es un ejemplo de la evolución que ha tenido el manejo de pacientes con tórax inestable. Inicialmente, se sometían a manejo quirúrgico mediante cerclaje con alambre; luego, a fijación con materiales de osteosíntesis para cirugía maxilofacial. En la actualidad, el $100 \%$ de los pacientes se somete a fijación con materiales de titanio, cuyo beneficio ha sido comprobado a nivel mundial; los nuevos dispositivos de fijación son visualizados en estudios de imagen, lo que los convierte en piedra angular en la evolución del tratamiento de pacientes con tórax inestable. Nuestros resultados se comparan positivamente con los reportados en la literatura moderna mundial.

\section{REFERENCIAS}

1. Pettiford BL, Luketich JD, Landreneau RJ. The management of flail chest. Thorac Surg Clin. 2007; 17: 25-33.

2. Miller LA. Chest wall, lung, and pleural space trauma. Radiol Clin North Am. 2006; 44: 213-224, viii.

3. Fowler TT, Taylor BC, Bellino MJ, Althausen PL. Surgical treatment of flail chest and rib fractures. J Am Acad Orthop Surg. 2014; 22: 751-760.

4. Bastos R, Calhoon JH, Baisden CE. Flail chest and pulmonary contusion. Semin Thorac Cardiovasc Surg. 2008; 20: 39-45.

5. Paydar S, Mousavi SM, Niakan H, Abbasi HR, Bolandparvaz S. Appropriate management of flail chest needs proper injury classification. J Am Coll Surg. 2012; 215: 743-744.

6. DeFreest L, Tafen M, Bhakta A, Ata A, Martone S, Glotzer $\mathrm{O}$, et al. Open reduction and internal fixation of rib fractures in polytrauma patients with flail chest. Am J Surg. 2016; 211: 761-767.

7. Sarani B, Schulte L, Diaz JJ. Pitfalls associated with open reduction and internal fixation of fractured ribs. Injury. 2015; 46: 2335-2340.

8. Moreno-De La Santa Barajas P, Polo-Otero MD, Delgado Sánchez-Gracián C, Lozano-Gómez M, Toscano-Novella A, Calatayud Moscoso-Del Prado J, et al. Surgical fixation of rib fractures with clips and titanium bars (STRATOS System). Preliminary experience. Cir Esp. 2010; 88: 180-186.

9. Bemelman M, van Baal M, Yuan JZ, Leenen L. The role of minimally invasive plate osteosynthesis in rib fixation: a review. Korean J Thorac Cardiovasc Surg. 2016; 49: 1-8.

10. Tanaka H, Yukioka T, Yamaguti Y, Shimizu S, Goto $\mathrm{H}$, Matsuda $\mathrm{H}$, et al. Surgical stabilization of internal pneumatic stabilization? A prospective randomized study of management of severe flail chest patients. J Trauma. 2002; 52: 727-732; discussion 732 .

11. Leinicke JA, Elmore L, Freeman BD, Colditz GA. Operative management of rib fractures in the setting of flail chest: a systematic review and meta-analysis. Ann Surg. 2013; 258: 914-921.

12. Jayle CP, Allain G, Ingrand P, Laksiri L, Bonnin E, HajjChahine J, et al. Flail chest in polytraumatized patients: surgical fixation using Stracos reduces ventilator time and hospital stay. Biomed Res Int. 2015; 2015: 624723.

13. Ahmed Z, Mohyuddin Z. Management of flail chest injury: internal fixation versus endotracheal intubation and ventilation. J Thorac Cardiovasc Surg. 1995; 110: 1676-1680.

14. Lafferty PM, Anavian J, Will RE, Cole PA. Operative treatment of chest wall injuries: indications, technique, and outcomes. J Bone Joint Surg Am. 2011; 93: 97-110.

15. Fitzpatrick DC, Denard PJ, Phelan D, Long WB, Madey SM, Bottlang M. Operative stabilization of flail chest injuries: review of literature and fixation options. Eur J Trauma Emerg Surg. 2010; 36: 427-433.

16. López-Bascopé A. Cambios históricos ó necesarios en la anestesiología actual. Anestesia en México. 2009; 21: 86-88.

17. Nirula R, Diaz JJ Jr, Trunkey DD, Mayberry JC. Rib fracture repair: indications, technical issues, and future directions. World J Surg. 2009; 33: 14-22.

18. Velmahos GC, Vassiliu P, Chan LS, Murray JA, Berne TV, Demetriades D. Influence of flail chest on outcome among patients with severe thoracic cage trauma. Int Surg. 2002; 87: 240-244.

19. Voggenreiter G, Neudeck F, Aufmkolk M, Obertacke U, Schmit-Neuerburg KP. Operative chest wall stabilization in flail chest - outcomes of patients with or without pulmonary contusion. J Am Coll Surg. 1998; 187: 130-138.

20. Tanaka H, Tajimi K, Endoh Y, Kobayashi K. Pneumatic stabilization for flail chest injury: an 11-year study. Surg Today. 2001; 31: 12-17.

21. Karlo CA, Stolzmann P, Do RK, Alkadhi H. Computed tomography of the spleen: how to interpret the hypodense lesion. Insights Imaging. 2013; 4: 65-76.

22. Slobogean GP, MacPherson CA, Sun T, Pelletier ME, Hameed SM. Surgical fixation vs. nonoperative management of flail chest: a meta-analysis. J Am Coll Surg. 2013; 216: 302-11.e1.

Correspondencia:

Dr. Agustín Luna-Tovar

Hospital Ángeles Mocel

Gelati Núm. 29, consultorio 209,

Col. San Miguel Chapultepec, 11850,

Del. Miguel Hidalgo, CDMX.

Tel: (55) 5615-0057

E-mail: agustinlunat@yahoo.com.mx 